\title{
Control design of an electro-pneumatic gearbox actuator
}

\author{
$1^{\text {st }}$ Adam Szabo \\ Department of Control for Transportation \\ and Vehicle Systems \\ Budapest University of Technology and Economics \\ Budapest, Hungary \\ szabo.adam@mail.bme.hu
}

\author{
$4^{\text {th }}$ Szilard Aradi \\ Department of Control for Transportation \\ and Vehicle Systems \\ Budapest University of Technology and Economics \\ Budapest, Hungary \\ aradi.szilard@mail.bme.hu
}

\begin{abstract}
The paper deals with the model-based control of an electro-pneumatic gearbox actuator. Electro-pneumatic systems have many advantages, such as long lifetime and high operational safety, but their nonlinear behavior makes predicting and controlling them difficult. The objective of the research is to design an optimal control of an electro-pneumatic gearbox actuator. In order to control a nonlinear system with linear control methods a multi-state state-space representation of the system is presented. Based on a preliminary comparison an open-loop, a PID, and a linear quadratic controller were chosen for development. The developed controllers were tested in a Model in The Loop environment, and their performances were compared based on previously defined controlling requirements. Both the open-loop and the PID controller were able to meet the given requirements. The open-loop controller has high parameter sensitivity and relative low performances in case of collision speed and shift time, while the PID controller has the highest number of solenoid valve actuations. The linear quadratic controller has good overall performance in case of neutral to gear changes, however it cannot stabilize the system in neutral position within the required time, which can be a consequence of the given simplifications. With respect to the results of the Model in the Loop tests two suggestions were made regarding the applicable controller.
\end{abstract}

Index Terms-Pneumatic actuators, Control design, Position control, PD control

\section{INTRODUCTION}

Pneumatic actuators use the compressed energy of the compressed gas to achieve force transmission and to produce reciprocating linear motion. Systems driven by pneumatic or electro-pneumatic actuators are widely used in industrial applications, since they have lower specific weight and higher power density than an equivalent electro-mechanic actuator,

*EFOP-3.6.3-VEKOP-16-2017-00001: Talent management in autonomous vehicle control technologies- The Project is supported by the Hungarian Government and co-financed by the European Social Fund

* The research is supported by the Magyar Automuszaki Felsooktatasert Alapitvany. and they are applicable on a wider temperature domain than the hydraulic actuators. There is an unlimited supply of air to be compressed, while the exhausted air do not need to be collected, which means fluid return lines are unnecessary. The compressed air can be easily stored, and transported, while these type of actuators are easily maintainable, they have long lifetime and high operational safety. They have a wide range of applications in the field of robotics, automation and manufacturing. A review of the industrial application of pneumatic systems can be found in [1].

Many of recent studies focuses on the modeling and control of pneumatic muscle actuators (PMA), such as [2], [3] and [4]. They are one of the most promising actuators for applications that require greater proximity between the humans and the robots. While researches connected to the vehicle industry focus on the single- and double acting pneumatic cylinders. These are commonly used in systems, like exhaust gas recirculation, air brake systems [5], turbocharger applications [6], electro-pneumatic clutch [7] and gearbox actuator systems [8].

While the electro-pneumatic systems have many advantages, their nonlinear behavior makes predicting and controlling them difficult. [9] In literature one of the proposed methods is PID control, which should be enhanced through gain scheduling or it should be used in cascaded control [10]. Another control methods are the linear quadratic (LQ) control [8], which provides optimal control solution for a given cost function, the sliding mode control [11] and H-infinity method [12]. Further applicable controllers are the adaptive controllers [13], the fuzzy-based controllers [14] and the Neural Network based controllers, such as [15], [16] and [17].

The objective of the research is to design different concepts of model-based controllers, which can assure the optimal position control of a given electro-pneumatic actuator, and to find an optimal control strategy, which can meet the contradictory requirements of the system. 
The paper is organized as follows: Section II describes the modeled actuator, Section III shows the state-space representation of the system. Section IV presents the performances of the developed controllers, Section V shows some conclusion remarks.

\section{SYSTEM DESCRIPTION}

The modeled actuator is used for the automated manual transmission of a heavy duty vehicles. Its function is to shift the proper gear inside a previously selected lane or to set the gearbox to neutral.

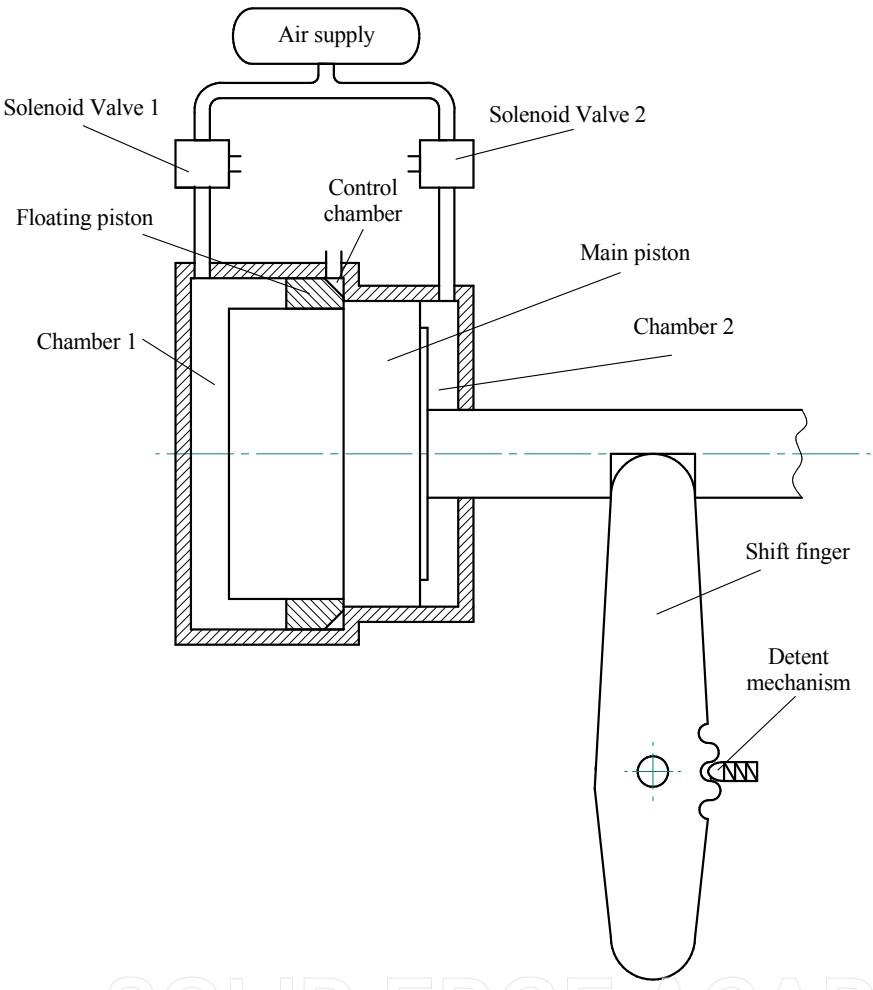

Fig. 1. Simplified layout of the gearbox actuator

The pneumatic cylinder is driven by two 3-way 2-position solenoid valves. In energized state the solenoid valves connect the chambers to the supply pressure, while in released state they connect them to the ambient pressure. There are two working chambers and a control chamber distinguished inside the cylinder. Both Chamber 1 and Chamber 2 are connected to a single solenoid valve which serves both input and output purposes, while the control chamber serves as an air spring with no solenoid valve connected to its only port. The layout of the modeled system can be seen in Figure 1.

To shift between different gears the main piston actuates the shift finger and through it the gear shift linkage and the synchronizer sleeve. It has three dedicated positions: two gears (High and Low end positions of the piston) and Neutral position. Its movement is generated by the opposing pressure forces inside the working chambers. The function of the floating piston is to act against the movement of the main piston through collision and to tune the volume of the control chamber.

\section{MOdEL DESCRIPTION}

To control the nonlinear system with linear methods, a multi-state state-space representation of the modeled system is presented. Since the modeled system shows input-affine properties, the representation can take the following form:

$$
\begin{gathered}
\underline{\dot{x}}=f(\underline{x}, \underline{d}, r)+\sum_{i=1}^{m} \underline{g}_{i}(\underline{x}, \underline{d}, r) u_{i} \\
\underline{y}=h(\underline{x}, \underline{d}, \underline{u}, r)
\end{gathered}
$$

where $x$ is the state vector, $y$ is the output vector, $u$ is the input vector, $d$ is the disturbance vector and $r$ is the hybrid mode mapping. The state matrices can be obtained by linearization, as it is shown:

$$
\begin{gathered}
A=\left.\frac{\partial f(\underline{x}, \underline{d}, r)}{\partial \underline{x}}\right|_{\substack{x=x_{0} \\
u=0}}, B=\left[\underline{g}_{1}, \underline{g}_{2}\right] \\
C=\left.\frac{\partial h(\underline{x}, \underline{d}, \underline{u}, r)}{\partial \underline{x}}\right|_{\substack{x=x_{0} \\
u=0}}, D=\left.\frac{\partial h(\underline{x}, \underline{d}, \underline{u}, r)}{\partial \underline{u}}\right|_{x=x_{0}}
\end{gathered}
$$

where $A$ is the state matrix, $B$ is the input matrix, $C$ is the output matrix, and $D$ is the feedthrough matrix.

The nonlinear model contains unmanageable number of hybrid states, therefore it had to be simplified for control design purposes. Compared to the nonlinear model the following simplifications were made:

- the solenoid valve models are substituted with their mass flow rates

- Detent mechanism is disregarded

- Contact forces are disregarded

- Control chamber pressure is assumed to be equal to the ambient pressure

- Heat transfer is neglected

- Coulomb-friction is neglected

- based on the main piston position, the floating piston is either at neutral position, or it is assumed to move together with the main piston

With the given simplifications the operation domain of the actuator can be described by two hybrid states, based on the position of the main piston and by two hybrid states for each solenoid valves, based on their input signals. The state vectors of the state-space representation are written as follows:

$$
\underline{x}=\left[\begin{array}{c}
p_{c h 1} \\
p_{c h 2} \\
x_{M P} \\
v_{M P}
\end{array}\right] \underline{y}=\left[\begin{array}{c}
p_{c h 1} \\
p_{c h 2} \\
x_{M P}
\end{array}\right] \underline{u}=\left[\begin{array}{c}
\dot{m}_{c h 1} \\
\dot{m}_{c h 2}
\end{array}\right] \underline{d}=\left[\begin{array}{c}
p_{a m b} \\
T_{a m b} \\
p_{s u p} \\
T_{s u p}
\end{array}\right]
$$

where $p$ is the pressure, $m$ is the air mass, $T$ is the temperature, $x$ is the position, amb refers to ambient, sup 
refers to supply, ch 1 refers to Chamber 1 , ch 2 refers to Chamber 2 and $M P$ refers to main piston.

With the given state vectors, the state-space representation takes the following form, where $\mathrm{f}, g_{1}, g_{2}$ and h vectors are the function of $\underline{x}, \underline{d}$ and $\mathrm{r}$ :

$$
\begin{gathered}
f=\left[\begin{array}{c}
\frac{-\kappa_{a i r} p_{c h 1} \dot{V}_{c h 1}}{V_{c h 1}} \\
\frac{-\kappa_{a i r} p_{c h 2} \dot{V}_{c h 2}}{V_{c h 2}} \\
v_{M P} \\
\frac{\sum F_{p}-d_{H} v_{M P}}{m_{M P}+m_{F P}}
\end{array}\right], h=\left[\begin{array}{c}
p_{c h 1} \\
p_{c h 2} \\
x_{M P}
\end{array}\right] \\
g_{1}=\left[\begin{array}{c}
\frac{\kappa_{a i r} R_{a i r} T_{i n w 1}}{V_{c h 1}} \\
0 \\
0 \\
0
\end{array}\right], g_{2}=\left[\begin{array}{c}
0 \\
\frac{\kappa_{a i r} R_{a i r} T_{i n w 2}}{V_{c h 2}} \\
0 \\
0
\end{array}\right]
\end{gathered}
$$

where $\kappa_{\text {air }}$ is the heat capacity ratio, $R_{\text {air }}$ is the gas constant for air, $V$ is the volume, $T_{i n w 1}$ and $T_{i n w 2}$ are the temperature of the air flowing to Chamber 1 and Chamber 2 .

A more detailed description of the nonlinear model can be found in [18].

\section{CONTROL DESIGN}

\section{A. Controlling aims}

To choose the applicable control methods, first the controlling goals have to be determined. To achieve the tasks described in Section II, the developed control has to meet different, contradictory requirements:

- Tracking of a reference position signal

- Maximum 80ms shift time

- Maximum 0,2m/s impact velocity at reaching the end positions of the cylinder

- Maximum $1 \mathrm{~mm}$ overshooting and amplitude when reaching neutral state

- Maximum 6 solenoid valve actuation

In terms of vehicle dynamics and comfort it is essential, to achieve the gear change as fast as possible. For this, high actuation force is required, however the high speed collision decreases the expected lifetime of the system and the comfort of the vehicle. A possible optimum between the two requirements is to divide the piston movement into two parts. In the first part of the movement the main task is the acceleration of the piston, therefore decrease the shift time, while in the second part the piston velocity should be decreased to minimize the collision speed or reduce the overshoot.

In case of impulse driven systems, like this, the optimal control would be a Pulse-Width Modulation method, however it is contradictory to the solenoid valve requirements.

Based on previous experiences it is assumable, that the discretization parameters of the solenoid valves has a higher effect on the number of solenoid actuations, than the controller parameters.
TABLE I

PRELIMINARY COMPARISON OF THE APPLICABLE CONTROL METHODS

\begin{tabular}{|c|c|c|c|c|c|c|}
\hline & PID & LQ & SMC & MPC & $H_{i n f}$ & O-L \\
\hline DoI (1) & 4 & 3 & 2 & 2 & 1 & 5 \\
\hline CC (3) & 4 & 3 & 2 & 3 & 1 & 5 \\
\hline GCL (3) & 4 & 3 & 1 & 1 & 1 & 5 \\
\hline S (2) & 2 & 3 & 5 & 3 & 5 & 1 \\
\hline P (2) & 2 & 4 & 5 & 4 & 5 & 1 \\
\hline Total & 36 & 35 & 31 & 28 & 27 & 39 \\
\hline
\end{tabular}

\section{B. Applicable methods}

Based on the available literature, the following control methods could be used to achieve position control:

- Open-loop control (O-L)

- Linearization based control (such as PID and linear quadratic (LQ) controls)

- Sliding mode control (SMC)

- Model predictive control (MPC)

- H-infinity control $\left(H_{i n f}\right)$

Considering the controlling aims and the dynamic properties of the system, it is obvious, that the chosen controller has to be able to handle multiple hybrid states. This can be done by using multi-state switching controls, or more complex control methods, such as the sliding mode control.

The mentioned controllers were compared based on their performance $(\mathrm{P})$, stability $(\mathrm{S})$, generated code length (GCL), computing cost (CC), and their difficulty of implementation (DoI). The features were rated on a scale of 1 to 5 , where 1 means the worst, while 5 the best rating. Then the different features were weighted. Since the controller has to be able to run on embedded systems, these features have the highest weight parameters, while the difficulty of implementation has the lowest weight parameter. The evaluation (see Table I) is based on literature and previous experiences.

Based on the results of Table I. the following methods were chosen:

- Open-loop control

- PID control

- Linear-quadratic control

\section{Test environment}

The implemented controllers were tested in a Model in the Loop environment, using the detailed, nonlinear model developed in [1]. The input signal of the environment is the reference position, which is processed by the controller. The control signals are the solenoid valve commands, while the system outputs are the position and velocity of the main piston. The layout of the test environment can be seen in Figure 2.

The simulation runs in $0.001 \mathrm{~ms}$, while the sample time of the controller is $1 \mathrm{~ms}$. All possible gear changes were tested with the developed controllers, but in the following sections only a part of these test cases will be shown.

\section{Open-loop control method}

In case of open-loop control, the control signal is one of four, previously determined solenoid valve sequence and it is 


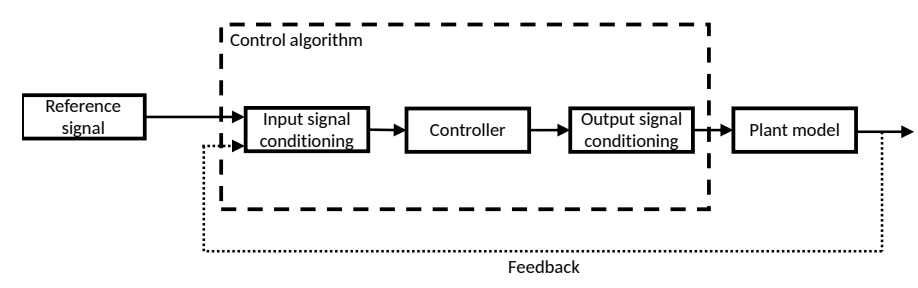

Fig. 2. Block scheme of the Model in the Loop environment

TABLE II

PERFORMANCES OF THE OPEN-LOOP CONTROLLER

\begin{tabular}{|l|c|c|c|c|}
\hline \multicolumn{1}{|c|}{ Open-loop controller } & N2L & L2N & N2H & H2N \\
\hline Simulation time [ms] & \multicolumn{5}{|c|}{23.71} \\
\hline Shift time [s] & 63 & 58 & 70 & 63 \\
\hline Collision speed [m/s] & 0.194 & - & 0.177 & - \\
\hline Maximum overshoot [mm] & - & 0.78 & - & 0 \\
\hline Number of solenoid actuations [pc.] & 2 & 2 & 2 & 2 \\
\hline
\end{tabular}

chosen with respect to the input signal. While determining the valve sequences, the following rules were taken into account:

- While switching into High or Low position, the collision speed should be decreased by loading the counter-side chamber

- While switching into Neutral, both of the chambers should be loaded at the same time

Three of the four gear changes can be accomplished by using the principles above, however in case of the Low to Neutral gear change the piston velocity is highly decreasing, therefore loading Chamber 2 has to be delayed compared to the loading of Chamber 1. This case can be seen in Fig 3. Figures showing the controller performances should be interpreted as follows: the first diagram shows the output signals of the presented controller (these are the solenoid valve commands), on the second diagram the chamber pressures are shown. The third diagram shows the input signals of the controller, which are the reference position and the actual position signal. The last diagram presents the piston velocity.

The results of the open-loop control can be found in Table II.

\section{E. PID control method}

First, a multi-state PID controller was used to control the system, but after further investigating the controlling aims and the dynamic properties of the system, the integrate term of the controller was terminated. The function of the integral term is to eliminate the residual error by adding a control effect due to the historic cumulative value of the error, but at the same time it decreases the stability and increases the overshoot of the system. The elimination of the residual error can be done by the detent mechanism, the additional dynamic assured by the integral term is not required, while increasing the overshoot is contradictory with the minimized collision speed and overshoot.
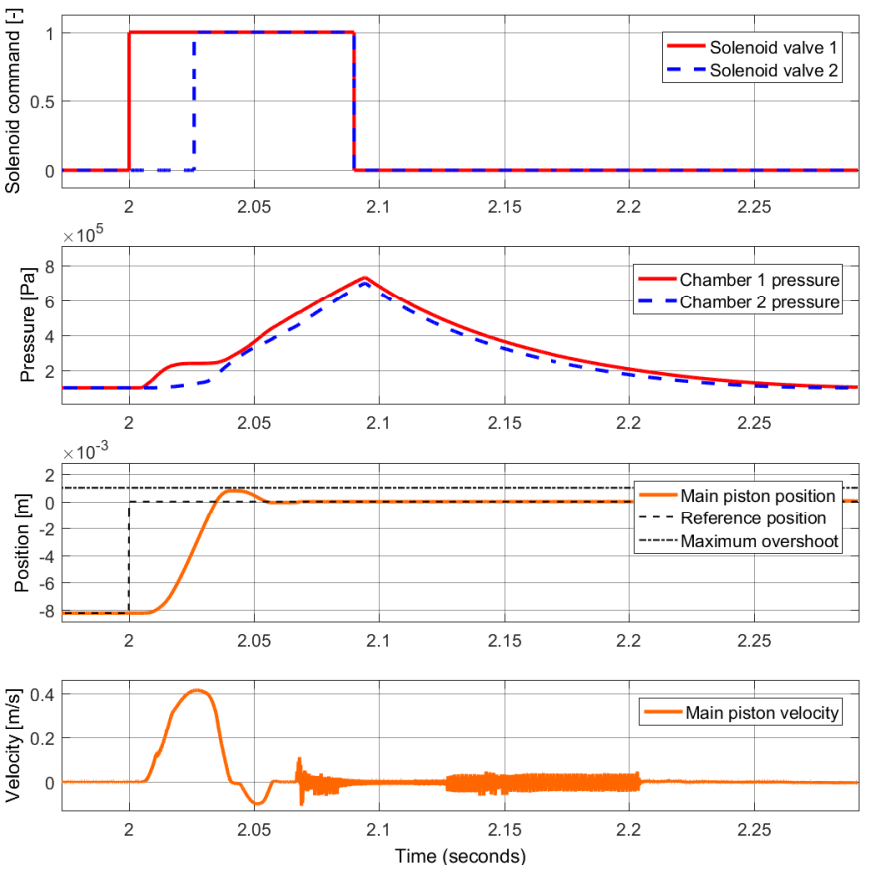

Fig. 3. Open-loop control - Low to Neutral gear change

In the controller, the input signal and the feedback signal (position of the main piston) are sampled with $1 \mathrm{~ms}$ to match a sample time suitable for embedded systems. If the calculated position error reaches a previously defined value, the controller intervenes, while under this value the detent mechanism has to be able to eliminate the error. There are four $\mathrm{P}$ and $\mathrm{D}$ parameter pairs, one for each possible request. The last part of the controller algorithm is to convert the output signal of the PD controller to solenoid valve commands. To prevent the back and forth activation between the solenoid valves there is in lane around zero, where both of the solenoid valves are inactive. Over this lane, Solenoid Valve 1, while under it Solenoid Valve 2 is activated. Because of the inertia of the system, the too short, but frequent solenoid valve commands are not effective, therefore the shortest enabled solenoid commands given by the controller are $5 \mathrm{~ms}$ long. This constraint has an other useful effect. Because of the lane of the control signal around zero, the controller would not be able to activate both valves at the same time, which would be useful in case of Gear to Neutral gear change. With this, it is possible that when reaching neutral with one of the valves, the control signal goes through the lane actuating the counter side valve, while the previously actuated valve is still active, because of the constraint. Since the simulation runs in $0.001 \mathrm{~ms}$ sample time, the output signal of the control logic also has to be sampled.

The parameter tuning of the PD controller was done by manually, since the widespread parameter tuning methods, such as the Ziegler-Nicholson method were not able to provide acceptable results.

The PD controller can be implemented without detailed knowledge of the controlled system, but its tuning was a 

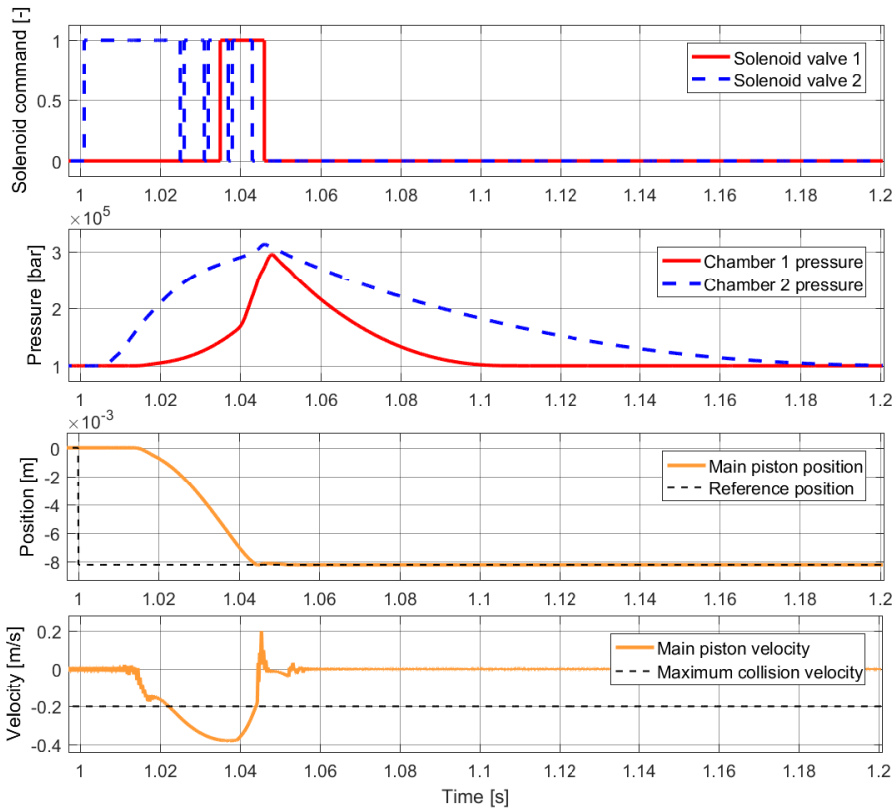

Fig. 4. PD control - Neutral to Low gear change

TABLE III

PERFORMANCES OF THE PD CONTROLLER

\begin{tabular}{|l|c|c|c|c|}
\hline \multicolumn{1}{|c|}{ PD controller } & N2L & L2N & N2H & H2N \\
\hline Simulation time [s] & \multicolumn{5}{c|}{25.33} \\
\hline Shift time [ms] & 44 & 49 & 59 & 56 \\
\hline Collision speed [m/s] & 0.179 & - & 0.162 & - \\
\hline Maximum overshoot [mm] & - & 0.36 & - & 0.99 \\
\hline Number of solenoid actuations [pc.] & 5 & 4 & 3 & 6 \\
\hline
\end{tabular}

complex task with respect to the contradictory controlling goals. The Neutral to High with PD controller can be seen in Fig 4.

The results of the PD controller development are summarized in Table III.

\section{F. Linear quadratic controller}

The processing of the input signals is the same as in case of the PD controller. In case of the linear quadratic controller, the state matrix and the input matrix are calculated according to the state space model given in III. The measured signals required to the matrix calculations are the outputs of the Model in the Loop environment. Based on the state matrix, input matrix and the weight matrices, the optimal gain matrix is calculated. The weight matrices were tuned manually. Since there is only position reference signal, the aim of the LQ controller development was to find an optimum between the weight of the position error and the cost of the input signals. The optimal gain matrix is then separated into vectors to determine the required mass flow rates by both of the solenoid valves. If the required mass flow rate exceeds a previously determined value, the solenoid valve is activated. Following this, the solenoid valve commands has to be sampled.
TABLE IV

PERFORMANCES OF THE LINEAR QUADRATIC CONTROLLER

\begin{tabular}{|l|c|c|c|c|}
\hline \multicolumn{1}{|c|}{ LQ controller } & N2L & L2N & N2H & H2N \\
\hline Simulation time [s] & \multicolumn{5}{|c|}{35.37} \\
\hline Shift time [ms] & 54 & 300 & 57 & 200 \\
\hline Collision speed [m/s] & 0.169 & - & 0.160 & - \\
\hline Maximum overshoot [mm] & - & 2.56 & - & 2.25 \\
\hline Number of solenoid actuations [pc.] & 1 & 2 & 1 & 3 \\
\hline
\end{tabular}

As an example, the Neutral to High gear change can be seen in Fig 5.
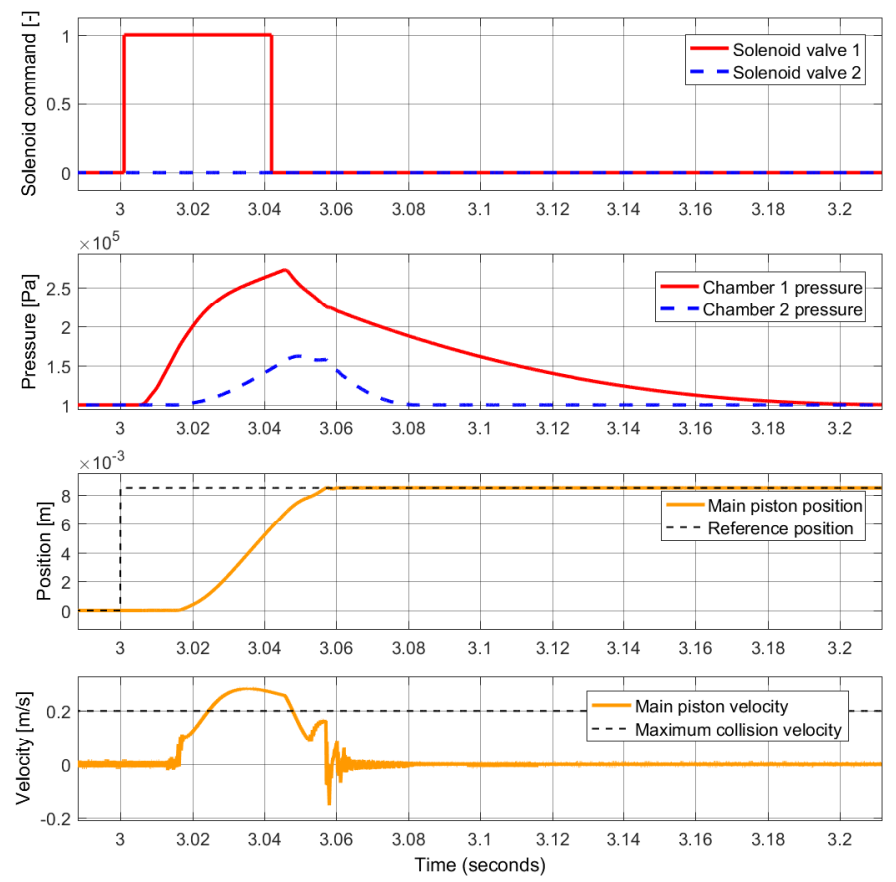

Fig. 5. Linear quadratic control - Neutral to High gear change

The results of the linear quadratic control development can be found in Table IV.

\section{CONCLUSIONS AND FUTURE WORK}

In this paper three different type of position control design has been presented through the application of a pneumatic gearbox actuator. The developed controllers were tested in Model in Loop Environment, and their performances were compared based on the given control aims and requirements.

The Open-loop controller satisfies the requirements given in Section IV in all test cases, but in its current form it cannot adapt to the changes of environmental and supply parameters.

The PD controller is also able to meet the given requirements, and it achieves faster gear changes than the Openloop controller, however it has the highest number of solenoid actuations in all test cases.

The linear quadratic controller has the highest simulation time and it is not able to meet the requirements in case of Gear to Neutral gear changes. This is presumably caused by the ignorance of the detent mechanism in the state space 
TABLE V

PERFORMANCES OF THE COMBINED OPEN-LOOP AND LQ CONTROLLER

\begin{tabular}{|l|c|c|c|c|}
\hline \multicolumn{1}{|c|}{ Combined controller } & N2L & L2N & N2H & H2N \\
\hline Shift time [ms] & 54 & 58 & 57 & 63 \\
\hline Collision speed [m/s] & 0.169 & - & 0.160 & - \\
\hline Maximum overshoot [mm] & - & 0.78 & - & 0 \\
\hline Number of solenoid actuations [pc.] & 1 & 2 & 1 & 2 \\
\hline
\end{tabular}

representation. However, it has the best results in case of Neutral to Gear shift. It has the smallest collision speeds, and the least amount of solenoid actuations.

Based on the results, two suggestions can be made regarding the applicable controller. The first one is the state based combination of the linear quadratic and the open-loop controller, which could utilize the advantages of both controllers. The performances of the combined controller are summarized in Table V. The second suggestion is the further improvement of the linear quadratic controller. Since the main function of the detent mechanism is to hold the actuator in the three dedicated position, by integrating a simplified model of it to the state-space representation, presumably the linear quadratic controller could be able to provide acceptable results in case of Gear to Neutral shifts.

Further research will focus on the implementation of a synchronizer mechanism to the nonlinear model, and on the parameter sensitivity analysis of the completed model. Researches on the external synchronization strategies of heavy duty vehicles can be found in literature, such as [19], [20] and [21]. However, as number of the AMT systems in the heavy duty vehicle market is increasing the manufacturers have to provide the manual transmission and the AMT systems on the same mechanical basis to meet the requirements of the cost-sensitive market. As a consequence of this, these AMT gearboxes has to be synchronized internally, instead of the application of countershaft brakes. The widespread gear change strategies cannot meet the requirements of the internally synchronized gearboxes, therefore new strategies have to be developed.

The developed models and controllers will be used to develop a cascade control loop, which can assure the position control of the actuator, while during synchronization it can reduce the stress appearing on the synchronizer elements.

\section{REFERENCES}

[1] D. Saravanakumar, B. Mohan, and T. Muthuramalingam, "A review on recent research trends in servo pneumatic positioning systems," Precision Engineering, vol. 49, no. Supplement C, pp. 481 - 492, 2017.

[2] A. Hošovský, J. Piteľ, K. Židek, M. Tóthová, J. árosi, and L. Cveticanin, "Dynamic characterization and simulation of two-link soft robot arm with pneumatic muscles," Mechanism and Machine Theory, vol. 103, no. Supplement C, pp. 98 - 116, 2016.

[3] M. Oliver-Salazar, D. Szwedowicz-Wasik, A. Blanco-Ortega, F. AguilarAcevedo, and R. Ruiz-Gonz'alez, "Characterization of pneumatic muscles and their use for the position control of a mechatronic finger," Mechatronics, vol. 42, no. Supplement C, pp. 25 - 40, 2017.

[4] M. D. Doumit and S. Pardoel, "Dynamic contraction behaviour of pneumatic artificial muscle," Mechanical Systems and Signal Processing, vol. 91, no. Supplement C, pp. 93 - 110, 2017.
[5] P. Karthikeyan, C. S. Chaitanya, N. J. Raju, and S. C. Subramanian, "Modelling an electropneumatic brake system for commercial vehicles," IET Electrical Systems in Transportation, vol. 1, no. 1, pp. 41-48, March 2011.

[6] A. Mehmood, S. Laghrouche, and M. E. Bagdouri, "Modeling identification and simulation of pneumatic actuator for vgt system," Sensors and Actuators A: Physical, vol. 165, no. 2, pp. 367 - 378, 2011.

[7] B. Szimandl and H. Németh, "Dynamic hybrid model of an electropneumatic clutch system," Mechatronics, vol. 23, no. 1, pp. 21 - 36, 2013.

[8] B. Szimandl and H. Németh, "Closed loop control of electro pneumatic gearbox actuator," in 2009 European Control Conference (ECC), Aug 2009, pp. 2554-2559.

[9] E. Palomares, A. Nieto, A. Morales, J. Chicharro, and P. Pintado, "Dynamic behaviour of pneumatic linear actuators," Mechatronics, vol. 45, no. Supplement C, pp. $37-48,2017$.

[10] A. Saleem, B. Taha, T. Tutunji, and A. Al-Qaisia, "Identification and cascade control of servo-pneumatic system using particle swarm optimization," Simulation Modelling Practice and Theory, vol. 52, no. Supplement C, pp. $164-179,2015$.

[11] B. Szimandl and H. Németh, "Sliding mode position control of an electro-pneumatic clutch system," IFAC Proceedings Volumes, vol. 46, no. 2, pp. 707 - 712, 2013, 5th IFAC Symposium on System Structure and Control.

[12] B. Szimandl and H. Németh, "Robust servo control design for an electropneumatic clutch system using the $\mathrm{H} \infty$; method," in 2014 IEEE/ASME 10th International Conference on Mechatronic and Embedded Systems and Applications (MESA), Sept 2014, pp. 1-6.

[13] Y.-T. Liu, T.-T. Kung, K.-M. Chang, and S.-Y. Chen, "Observer-based adaptive sliding mode control for pneumatic servo system," Precision Engineering, vol. 37, no. 3, pp. 522 - 530, 2013.

[14] T. Nuchkrua and T. Leephakpreeda, "Fuzzy self-tuning pid control of hydrogen-driven pneumatic artificial muscle actuator," Journal of Bionic Engineering, vol. 10, no. 3, pp. $329-340,2013$.

[15] N. N. Son, C. V. Kien, and H. P. H. Anh, "A novel adaptive feedforwardpid controller of a scara parallel robot using pneumatic artificial muscle actuator based on neural network and modified differential evolution algorithm," Robotics and Autonomous Systems, vol. 96, no. Supplement C, pp. $65-80,2017$.

[16] C.-J. Chiang and Y.-C. Chen, "Neural network fuzzy sliding mode control of pneumatic muscle actuators," Engineering Applications of Artificial Intelligence, vol. 65, no. Supplement C, pp. 68 - 86, 2017.

[17] L. XiaoJun, Z. ChengRui, L. HongBin, and W. XinLiang, "Electronic pneumatic clutch control of the heavy truck based on neural network pid," in 2006 IEEE International Conference on Vehicular Electronics and Safety, Dec 2006, pp. 232-235.

[18] A. Szabó, T. Bécsi, P. Gáspár, and Sz. Aradi, "Control oriented modeling of an electro-pneumatic gearbox actuator," European Control Conference, Submitted, 2017.

[19] Bóka, G., Márialigeti, J., Lovas, L. and Trencséni, B. External synchronization strategies for automated mechanical transmissions with face dog clutch and countershaft brake. Buletin Stiintific-Universitatea Din Baia Mare-Seria C Fascicula Organe De Masini Tribologie Constructii De Masini XXIII: pp. 75-80. (2009)

[20] Bóka, G., Trencséni, B. and Németh, H. Look-up Based Synchronizer Logic for the Effective Actuation of the Countershaft Brake in a Heavy duty AMT with Face Dog Clutch. In: Proceedings of FISITA World Automotive Congress 2010. Budapest, Hungary, GTE, pp. 1-6. Paper F2010C097 (2010), granted with 'Outstanding Paper Award'

[21] Bóka, G., Lovas, L., Márialigeti, J. and Trencséni, B. Engagement capability of face-dog clutches on heavy duty automated mechanical transmissions with countershaft brake. Proc. IMechE, Part D: J. Automobile Engineering, 224 (D9), pp. 1125-1139. DOI 10.1243/09544070JAUTO1435 (2010) 